Cahiers de recherches médiévales

Entre Moyen Âge et Renaissance : continuités et ruptures. L'héroïque

\title{
L’idée de « deux cités »
}

L'influence de saint Augustin sur Christine de Pizan

\section{Xiangyun Zhang}

\section{(2) OpenEdition}

Journals

Édition électronique

URL : https://journals.openedition.org/crm/2082

DOI : $10.4000 / \mathrm{crm} .2082$

ISSN : $1955-2424$

Éditeur

Honoré Champion

Édition imprimée

Date de publication : 15 avril 2004

Pagination : 121-132

ISSN : 1272-9752

Référence électronique

Xiangyun Zhang, «L'idée de " deux cités » », Cahiers de recherches médiévales [En ligne], 11 spécial |

2004, mis en ligne le 18 octobre 2007, consulté le 15 décembre 2022. URL : http://

journals.openedition.org/crm/2082 ; DOI : https://doi.org/10.4000/crm.2082

Ce document a été généré automatiquement le 15 décembre 2022.

Tous droits réservés 


\title{
L'idée de « deux cités »
}

\author{
L'influence de saint Augustin sur Christine de Pizan
}

\section{Xiangyun Zhang}

1 L'influence de saint Augustin sur Christine de Pizan est évidente à première vue. D'abord, il l'a inspirée avec le titre de son chef d'œuvre : le De Civitate Dei, Cité de Dieu d'après la plus ancienne traduction française de Raoul de Presles ${ }^{1}$, puisque Christine a nommé son livre le plus célèbre, La Cité des Dames, ouvrage rédigé dix siècles après celui de son prédécesseur. Ensuite, en prenant l'exemple de saint Augustin pour qui l'imputation faite aux chrétiens du désastre du sac de Rome par les hordes d'Alaric le 24 août 410 est devenue une occasion d'écrire La Cité de Dieu ${ }^{2}$, la femme écrivain a aussi profité d'un événement historique et littéraire, le Débat sur le Roman de la Rose, pour produire son œuvre ${ }^{3}$. Et finalement, la méthode polémique du Père de l'Église a sans doute servi de modèle à Christine pour bâtir une cité des dames à partir de sa fondation : chacune des questions que Christine la narratrice pose aux trois Dames du Ciel, Raison, Justice et Droiture, représente une opinion négative des hommes sur les femmes, et chaque exemple d'une ou des femmes vertueuses que les trois Dames utilisent pour répliquer à ces opinions symbolise un morceau de brique que Christine ajoute à la construction de sa cité allégorique. Les deux ouvrages, ceux de saint Augustin et de Christine de Pizan, ressortissent donc au même genre ${ }^{4}$.

2 Mais pour l'auteur du De Civitate Dei, répondre aux détracteurs du christianisme ne représente qu'un des moyens pour défendre cette religion et la réfutation des opinions erronées et hostiles à la religion chrétienne n'occupe qu'une partie de ce chef d'œuvre ${ }^{5}$. Saint Augustin avait en effet élaboré, pour son travail, un plan qui dépasse de loin celui d'une simple œuvre de circonstance parce qu'il s'y efforce non seulement d'établir l'honneur de l'Église, mais surtout d'inciter les païens à se convertir au christianisme afin d'augmenter la population de la cité de Dieu: "C'est à cette partie que nous te convions; viens, nous t'y exhortons, viens grossir le nombre de ses citoyens: leur asiles, à eux, c'est, si j'ose dire, la véritable rémission des péchés ${ }^{6}$. Et c'est pour indiquer le chemin amenant au vrai Dieu aux païens aussi bien qu'aux chrétiens qu'il leur donne une leçon sur les « deux cités $»^{7}$ dans le De Civitate Dei. Selon la doctrine de l'évêque d'Hippone, le monde créé par Dieu est une cité dont tous les êtres humains 
sont naturellement citoyens - c'est la cité de Dieu. Sous le toit de cette cité les habitants sont divisés en deux groupes selon leurs différents amours. Ceux qui aiment mieux le Seigneur qu'eux-mêmes appartiennent à la cité céleste, ou la cité de Dieu; ceux qui aiment mieux les intérêts personnels que notre Sauveur sont classés à la cité terrestre, ou cité du Diable. Mais, pour chaque citoyen, cette division n'est définitive qu'au moment du Jugement final ; autrement dit, les portes des deux cités restent toujours ouvertes à tout le monde ${ }^{8}:$ «Deux amours ont fait deux cités : l'amour de soi jusqu'au mépris de Dieu a fait la cité terrestre ; l'amour de Dieu jusqu'au mépris de soi a fait la cité céleste $»^{9}$.

3 Sur le plan de son travail à elle, Christine de Pizan a aussi suivi les pas du Père de l'Église puisqu'elle n'a pas laissé sécher sa plume après la rédaction du Livre de la Cité des Dames. La fin de ce dernier livre signale bien que la construction de cette communauté des femmes, bien que matériellement achevée, sera continuée dans un sens moral et intellectuel. Cela se voit dans le fait qu'après avoir installé, à leurs places appropriées dans tous les domaines de la cité, les femmes de haute réputation tirées des histoires et de la mythologie ainsi que les saintes ayant à leur tête la Vierge Marie, Christine s'adresse, dans le dernier chapitre de La Cité des Dames, non aux habitantes de la cité, mais aux femmes encore exclues de la citadelle. À ces femmes de toutes conditions sociales et de tous états matrimoniaux, elle montre le chemin à suivre pour arriver à la cité des Dames. Son appel aux femmes d'imperfection représente le but de son nouveau projet qui s'identifie alors à celui de saint Augustin : «Et ainsi vous plaise, mes tres redoubtees, par les vertus attraire et fouyr les vices, acroistre et mouteplier nostre cité, vous resjouyr et bien faire $»^{10}$. Cette tâche d'accroître et de multiplier les habitantes de la communauté des femmes que l'auteur de La Cité des Dames se donne ici, elle va l'accomplir dans la composition d'un autre livre.

4 L'étude sur l'idée de " deux cités » chez Christine de Pizan est basée avant tout sur un fait déjà largement reconnu par des critiques spécialisés de cet écrivain ${ }^{11}$ : Le Livre de la Cité des Dames et Le Livre des Trois Vertus, connu aussi sous le titre du Trésor de la Cité des Dames, doivent être considérés comme les deux volumes d'une même œuvre sous tous les aspects $^{12}$. Du point de vue chronologique les deux livres sont composés approximativement en même temps, entre 1404 et 1405 ; du point de vue textuel, la réapparition des trois Filles de Dieu dans Le Livre des Trois Vertus pour diriger Christine la narratrice dans la construction d'un édifice initiée dans La Cité, ainsi que la continuation du premier livre dans le deuxième que l'auteur annonce à la fin du Livre de la Cité des Dames ${ }^{13}$ établissent évidemment un rapport intertextuel qui lie étroitement les deux livres ensemble ; et finalement du point de vue thématique, les deux ouvrages, en tant qu'un seul monument allégorique, sont établis sur le même projet de construire et de renforcer la communauté des femmes afin de faire éblouir la gloire du sexe féminin.

5 L'unité des deux textes de Christine de Pizan permet de voir d'abord une analogie de structure entre les œuvres des deux auteurs puisqu'en réalité la Cité de Dieu est aussi composée de deux parties. Avant même que son œuvre ait vu le jour, saint Augustin a exprimé son intention de diviser sa Cité de Dieu en deux volumes dans sa suggestion au prêtre africain et « publiciste » Firmus :

Quaterniones sunt XXII quos in unum corpus redigere multum est. Et si duos uis codices fieri, ita diuidendi sunt ut decem libros habeat unus, alius duodecim. Decem quippe illis uanitates refutatae sunt impiorum, reliquis autem demonstrata atque defensa est nostre religio, quamuis et in illis hoc factum sit ubi oportunius fuit et in istis illud ${ }^{14}$. 
Il y a vingt-deux cahiers ; c'est beaucoup pour les réunir en un seul tout. Si tu veux en faire deux volumes, il convient de les grouper de telle manière que l'un comporte dix livres, l'autre douze. En effet, dans les dix premiers livres je réfute la vanité des impies, dans les autres j'expose et je défends notre religion, bien que, chaque fois que cela me parut opportun, je propose également cette défense dans les premiers livres et je poursuis cette réfutation dans les derniers ${ }^{15}$.

Il serait trop limité de considérer le De Civitate Dei comme un simple pamphlet polémique écrit pour répliquer aux attaques des adversaires ainsi que pour justifier sa religion devant la responsabilité de la chute de Rome que les païens avaient rejetée sur les chrétiens. Le besoin de produire un tel ouvrage s'imposait à lui depuis longtemps ${ }^{16}$ avant cet événement :

L'Église est alors attaquée sur tous les fronts à la fois : on lui reproche sa foi en la survie et en la résurrection de la chair ; on combat la divinité de Jésus-Christ et sa naissance virginale; on met en doute la véracité des Écritures; on lui fait grief d'une morale trop rigoureuse ; il est impossible d'être en même temps bon chrétien et bon citoyen $[. . .]^{17}$.

7 Devant toutes ces attaques, saint Augustin était bien conscient que l'accusation faite aux chrétiens du malheur du temps n'était qu'une apparence de toutes les objections qui se dirigeaient contre le christianisme, et que ce débat actuel sur la cause de la ruine de l'Empire Romain n'était qu'une prolongation de la dispute permanente entre les « deux cités ». Dans ce cas-là, une riposte était nécessaire mais insuffisante pour faire apparaître la vérité de la religion chrétienne. En rédigeant le De Civitate Dei, le Père de l'Église a donc dessiné une perspective gigantesque de «[...] donner à la chrétienté du $\mathrm{V}^{\mathrm{e}}$ siècle, et à tous ceux du monde païen pour qui cette chrétienté posait un problème, une sorte de "somme de la culture chrétienne» qui permettrait aux chrétiens de s'instruire, et aux païens ébranlés dans leurs convictions par les récents événements politiques, de se convertir au christianisme $»^{18}$. Il est important de comprendre que cette « somme de la culture chrétienne » comprend en effet l'origine, le développement ainsi que la fin des mondes terrestre et céleste ${ }^{19}$. Il s'agit alors de l'idéologie de « deux cités » sur laquelle saint Augustin allait composer les douze derniers livres de la Cité de Dieu.

8 Cette période difficile qu'a affrontée l'évêque d'Hippone nous rappelle le début du Livre de la Cité des Dames où Christine de Pizan met sa narratrice dans une situation semblable. La lecture de Mathéolus la jette dans un état où elle se sent accablée des paroles venimeuses des hommes contre les femmes et désespérée de son être féminin ainsi que de la façon dont la femme est vue à son époque. Sa propre conduite en tant que femme et celle des autres qu'elle connaît ne peuvent que la pousser à se demander pourquoi tant d'hommes portent de faux jugements sur les femmes :

Et nom mie seulement un ou deux ne cestuy Matheolus, qui entre les livres n'a aucune reputatcion et qui traitte en maniere de trufferie, mais generaument aucques en tous traittiez philosophes, pouettes, tous orateurs desquelz les noms seroit longue chose, semble que tous parlent par une meismes bouche et tous accordent une semblable conclusion, determinant les meurs femenins enclins et plains de tous les vices ${ }^{20}$.

9 L'essence de la femme était calomniée ; et devant ces attaques violentes venant de tous côtés, comme son prédécesseur, Christine de Pizan se sentait responsable de rétablir l'image de la femme déformée par des hommes tels qu'Ovidius, Mathéolus et Jean de Meun en particulier, de montrer la vraie nature du sexe féminin, et de lui rendre la justice. Ce sont les trois représentantes de Dieu, touchées par la situation, qui sont 
arrivées pour consoler Christine, pour la tirer de son désarroi, et surtout pour réviser l'histoire de la culture féminine avec elle - il s'agit aussi d'une " somme de la culture ", mais féminine. Le fait que Christine la narratrice pouvait obtenir « la prerogative entre les femmes de faire et bastir la Cité des Dames ${ }^{21}$ montre bien que Christine l'écrivain était consciente que les misogynes avaient agi contre la volonté divine lorsqu'ils avaient étiqueté toutes les femmes comme naturellement vicieuses : «Et en conclusion de tout, je determinoye que ville chose fist Dieux quant il fourma femme, en m'esmerveillant comment si digne ouvri[e]r daigna oncques faire tant abominable ouvrage qui est vaissel, au dit d'iceulx, si comme le retrait et herberge de tous maulx et de tous vices ${ }^{22}$. Mais Dieu ne saurait être vraiment tenu pour responsable d'une telle erreur et Christine avait sans doute l'intention d'établir l'autorité divine dans son livre aussi bien que dans la cité des Dames en opposant à l'avis des grands philosophes, poètes et moralistes, la volonté profonde de Dieu. De fait, la fondatrice de la cité des Dames est aussi le défenseur de la cité de Dieu. On comprend dès lors l'ironie de Christine au début de La Cité des Dames, lorsqu'elle laisse sa narratrice se lamenter sur Dieu pour ne l'avoir pas fait naître mâle ${ }^{23}$ : la division entre les «deux cités » se fait entre les vicieux et les vertueux plutôt qu'entre les hommes et les femmes.

Si l'idée de "deux cités " que Christine de Pizan hérite de son prédécesseur se manifeste dans sa conception du monde humain, on la voit à l'œuvre dans sa façon d'organiser la société des femmes. Comme l'auteur du De Civitate Dei, Christine a certainement compris et accepté l'idée de la cité dans la vie antique ${ }^{24}$. Construire la cité des dames, c'est unir les femmes derrière les mêmes murailles, les soumettre aux mêmes lois - celles de l'amour de Dieu et de la pratique des vertus - afin de mieux les protéger contre les ennemis communs. Mais le principe de la construction de cette communauté permet surtout de voir comment Christine de Pizan a adopté l'idée de "deux cités » développée et cristallisée par saint Augustin sur celle de la cité des anciens : "L'originalité de saint Augustin, [...] est d'avoir introduit, dans l'idée de cité, celle d'un amour en vertu duquel chaque homme peut librement choisir la cité à laquelle il appartient et d'avoir montré qu'il ne peut pas y avoir plus de deux amours, celui de Dieu et celui de soi » ${ }^{25}$. Dans Le Livre de la Cité des Dames, il ne peut être plus évident que l'auteur essaie d'établir la gloire des femmes. Mais cela ne devrait pas donner l'impression que Christine considère toutes les femmes comme vertueuses et qualifiées pour être citoyennes de cette cité. Elle ne prend pas une position extrême, comme ses adversaires le font, en réfutant les hommes qui pensaient que les femmes vertueuses étaient plus rares que le phénix ${ }^{26}$. C'est pourquoi, même avant que les travaux de la cité des Dames commencent, les trois Directrices de Christine la narratrice insistent déjà sur la persistance de l'édifice. Elles lui indiquent clairement que pour éviter l'écroulement de cette cité, comme ce qui était arrivé aux grandes cités de Troie, de Thèbes ainsi qu'au royaume d'Amazonie, la cité des Dames sera construite non pour toutes les femmes, mais seulement pour les méritantes: «[...] en laquelle n'abitera fors toutes dames de renommee et femmes dignes de loz : car a celles ou vertu ne sera trouvee, les murs de nostre cité seront forclos ${ }^{27}$. Les Déesses ont donc choisi les matériaux pour la construction de la cité allégorique de Christine : ce sont les vertus féminines, symbole de l'éternité.

11 De ce fait, comme le Père de l'Église, Christine de Pizan a également divisé la société des femmes en deux parties ou en «deux cités». Celles qui ont gagné honneur et réputation sont invitées à occuper une place dans la glorieuse cité des Dames, tandis 
que le reste des femmes sera enfermé dans la cage - qui est en réalité une autre image du même édifice - dans Le Livre des Trois Vertus. Mais que fait Christine de ces femmes qu'elle refuse d'admettre dans sa cité ? En seront-elles à jamais exclues ? Cette question nous permettra d'analyser comment l'auteur de La Cité des Dames a consciemment appliqué la structure mentale de "deux cités » dans la composition de ses deux livres. Pour saint Augustin, les « deux cités » sont à la fois séparées - puisqu'elles symbolisent respectivement le bien et le mal - et enchevêtrées - parce qu'il croit voir chez les adversaires une réserve de la cité de Dieu: "Qu'elle se souvienne pourtant que parmi ses ennemis mêmes se cachent de ses futurs citoyens, de peur qu'elle ne croie qu'il n'y a nul bénéfice à les supporter patiemment comme ennemis, jusqu'au jour où ils proclameront leur foi ${ }^{28}$. Et c'est justement pour convertir ces ennemis en citoyens de la cité de Dieu qu'il a composé la deuxième partie de cet ouvrage.

Ce qu'il importe de noter ici est que les ennemis de la cité de Dieu dont saint Augustin parle sont les païens qui blâment les chrétiens à cause du sac de Rome, tandis que pour Christine de Pizan, il y a deux sortes d'ennemis : les hommes qui calomnient les femmes et les femmes qui ne possèdent pas de qualités suffisantes pour être membres de la cité des Dames, puisque selon la définition des anciens pour la cité, tous ceux qui se trouvent en dehors de la cité sont des ennemis ${ }^{29}$. Ce sont bien sûr ces dernières que Christine essaie de recruter dans la cité des Dames. Loin d'avoir oublié ces femmes, Christine se hâte, malgré sa fatigue causée par la construction de la cité, d'établir une école des femmes. C'est évidemment pour corriger et perfectionner les écolières afin de les transformer en femmes vertueuses et donc citoyennes de la cité que Christine est forcée par les trois Déesses de reprendre la plume et de repartir au «champ des escriptures ». On arrive alors à comprendre qu'après l'établissement matériel de la communauté des femmes, la construction spirituelle commence à prendre la première importance pour la fondatrice de la cité des Dames. Bernard Ribémont nous montre, d'une manière figurative, l'unité des deux livres de Christine lorsqu'il appelle Le Livre des Trois Vertus une "véritable clé ouvrant la porte de la cité des dames ${ }^{30}$. Il nous explique en même temps pourquoi ce livre d'enseignement de l'école des femmes est appelé Le Trésor de la Cité des Dames: les femmes, sans exception, doivent acquérir les qualités vertueuses si elles veulent avoir leurs noms resplendir, comme des trésors, dans la cité des Dames.

13 Si la cité est faite pour les femmes d'honneur ${ }^{31}$, il semble contradictoire que, dans le prochain livre, les trois Filles Divines demandent à Christine la narratrice de jouer le rôle du « sage oiselleur " pour attraper les " oisillons » et les mettre dans la " cage », et lorsqu'elles exigent que la narratrice tende le « laz » dans tous les coins où les dames et "gereraument toutes femmes " ${ }^{32}$ pourraient passer. La " cage » est donc préparée non seulement pour les femmes non citoyennes de la cité, mais également pour les dames qui y sont déjà honorablement installées, y compris les dames de haut rang social. Il faut noter que Le Livre des Trois Vertus était en réalité écrit pour guider les pas de Marguerite, la Princesse de Bourgogne, fiancée au dauphin Charles de France à un âge très jeune $\mathrm{e}^{33}$; pourtant dans ce livre Christine établit des règles à suivre dans la vie de tous les jours pour les femmes de toutes conditions sociales, des hautes princesses jusqu'aux femmes de marchand et de paysan, et elle n'a même pas oublié les « femmes de fole vie $»^{34}$. Cela montre qu'elle va pratiquement enfermer toutes les femmes dans la "cage». Cette contradiction forme alors un autre moyen pour comprendre l'idée de " deux cités » que Christine de Pizan a empruntée à son prédécesseur : « Il y a d'ailleurs autre chose, et qui complique encore la distinction à établir entre les deux cités. Aussi 
longtemps qu'ils vivent ici-bas, les hommes appartiennent en effet à l'une et à l'autre, et ils visent tous également des biens matériels encore qu'ils ne poursuivent pas la même fin $»^{35}$. Saint Augustin se montre révolutionnaire en indiquant cette double appartenance inévitable à l'une cité ou à l'autre chez tous les êtres humains. Pour lui, l'homme est à tout moment libre de choisir sa "citoyenneté ", c'est-à-dire qu'il est toujours sujet au bien comme au mal. L'auteur de La Cité des Dames comprend certainement bien que cette dualité existe chez tout le monde, y compris les femmes, et c'est dans le but de diriger tous les membres de sa communauté vers le bien qu'elle décide de fonder une " université des femmes » dans Le Livre des Trois Vertus : «[...] que mon entendement ne y souffise, neanmoins, par pure et bonne intencion qui a ce me maine par le grant desir que j'ay de l'acroissement du bien et honneur de toute femme, grande, moyenne et petite $»^{36}$.

Est-ce par hasard que le "premier et principal enseignement» de cette école est : "d'aimer et craindre nostre Seigneur $\|^{37}$ ? La réponse à cette question ne peut qu'être négative. Une étude attentive de la première leçon dans Trois Vertus nous persuadera que les trois Dames, ou plutôt Christine, sont en train d'interpréter la doctrine de " deux cités », sujet primordial du De Civitate Dei ${ }^{38}$ :

Quel chose est il en ce monde plus plaisant ne tant delictable a ceulz qui desirent richeces mondaines que or et pierres precieuses? Mais ne leur porroyent mie pour tant si embelir que font vertus aux cuers qui desirent bien vivre, car de tant que vertus sont plus nobles que richeces mondaines. Et pour quoy sont elles plus nobles? Pour ce que elles durent sans fin et sont les tresors de l'ame qui est perpetuele, et les autres passent si comme fumee, de tant ceulz qui le goust en sentent et assaveurent les desirent ardemment plus que autre chose mondaine ne porroit estre desiree ${ }^{39}$.

Aimer soi-même ou aimer Dieu, cette « formule lapidaire » qui résume l'ouvrage entier de saint Augustin, Christine l'a clairement choisie comme base de son enseignement dans ce livre du miroir pour les femmes: "Si sera le fondement de nostre doctrine tout premierement sur l'amour et craintte de Nostre Seigneur, car cellui point est le principe et sapience dont toutes les aultres vertus yssent et deppendent $»^{40}$. Cette leçon de l'amour de Dieu commence au chapitre II du livre des Trois Vertus et elle continue jusqu'au chapitre VIII avant qu'elle se mette à donner des conseils spécifiques aux différentes femmes. Christine indique même deux façons concrètes d'aimer ou de servir le Seigneur qu'elle appelle les « deux voies qui meinent au ciel»: l'une est la «vie contemplative» et l'autre la «vie active $»^{41}$. La vie contemplative est l'état où une femme s'abandonne dans l'amour de Dieu de telle manière qu'elle oublie tout ce et tous ceux qui l'entourent; la vie active est un autre moyen d'aimer et de servir le Seigneur selon lequel la personne charitable doit, de temps en temps, rendre visite aux malades et aux pauvres; elle va essayer de les aider le mieux qu'elle le peut. On remarque facilement, si l'on continue à analyser chaque chapitre du Livre des Trois Vertus, que Christine de Pizan, à l'exemple de saint Augustin, essaie de montrer une telle réalité dans son livre : c'est dans la cité terrestre que tous les maux - l'orgueil, l'envie, et la médisance, etc. - prennent leurs racines parce que « La cité terrestre ne songe qu'à s'agrandir, à dominer ses ennemis, à acquérir gloire et puissance matérielles ${ }^{42}$. Christine fait remarquer quelquefois les mêmes problèmes dont saint Augustin fait la démonstration dans la seconde partie de la Cité de Dieu comme par exemple vivre selon la chair ou selon l'esprit ${ }^{43}$; la moralité du corps et le 
désir de la chair ${ }^{44}$; et l'existence terrestre se déroule inévitablement dans le malheur et la souffrance ${ }^{45}$, etc.

Une autre analogie s'établit sur ce point entre saint Augustin et son successeur français du Moyen Âge: les deux ont ajouté le genre didactique après la pratique du genre polémique dans leurs écrits. Le Trésor de la Cité des Dames est sans doute considéré comme un livre purement didactique, tandis que le De Civitate Dei n'est pas généralement vu dans ce genre. C'est parce que d'une part la première partie du livre qui est typiquement polémique cache l'évidence du genre de la deuxième, et d'autre part saint Augustin n'y emploie pas les termes de l'enseignement tels que leçon, école, et université, comme le fait Christine de Pizan dans son ouvrage. Pourtant, personne ne peut nier que le Père de l'Église voulait instruire la société de son époque. Il a écrit la Cité de Dieu pour les païens qui regardaient vers l'Église mais incapables de prendre la décision correcte, il l'a aussi écrite pour les chrétiens qui avaient toujours besoin d'être introduits plus profondément dans le mystère religieux et plus près du Seigneur : « On le voit, aucune contestation n'est possible sur le cadre général de l'œuvre : celle-ci est composée de deux parties, l'une, plus apologétique, en dix livres, se subdivisent ellemême en deux fois cinq livres ; l'autre, plus didactique, en douze livres, se subdivisant à son tour en trois fois quatre livres $»^{46}$.

17 C'est le genre didactique qui permet aux deux auteurs de rédiger leurs livres d'une manière réaliste. Christine de Pizan, dans la composition du Livre des Trois Vertus, descend des tours de la cité des Dames, cité que Charity Cannon Willard appelle " utopie féminine ${ }^{47}$, et se présente devant les femmes dans la société contemporaine ${ }^{48}$. En indiquant et en corrigeant les faiblesses des femmes, la narratrice enlève son masque de femme naïve qu'elle porte dans La Cité des Dames, elle s'identifie à la vraie image de l'auteur et devient alors professeur de l'« université des femmes» dans le deuxième livre. L'opinion critique de Jean-Claude Guy nous présente un saint Augustin réaliste et nous expliquerait probablement en même temps pourquoi la cité des Dames est la cité lorsque Christine réplique aux misogynes dans un livre et pourquoi elle devient la cage pour toutes les femmes dans un autre :

Dans la Démonstration ${ }^{49}$, le christianisme est présenté tel qu'il apparaît à l'intérieur de la foi et de la révélation, tel qu'il est en soi et pour soi ; dans la Réfutation, au contraire, il est présenté tel qu'il apparaît au païen, c'est-à-dire tel qu'il pose en face des fausses religions, en tant que seul capable de résoudre les problèmes que posent ces religions sans pouvoir y apporter de réponse ${ }^{50}$.

18 Il faut remarquer que chez les deux auteurs, l'enseignement qu'ils donnent - saint Augustin dans la deuxième partie du De Civitate Dei et Christine de Pizan dans Le Livre des Trois Vertus - démontre une stratégie pour réfuter leurs ennemis respectifs. Le Père de l'Église ne voulait pas se voir reprocher d'avoir renversé les idées d'autrui sans établir les siennes. Pour lui, « [...] la vérité du christianisme n'apparaitra, la cité de Dieu ne sera définitivement victorieuse du paganisme que lorsque ce dernier n'aura plus rien à revendiquer contre elle, mais verra au contraire dans la cité de Dieu la réalisation parfaite de ce vers quoi il croyait tendre, sans être capable d'y parvenir $"{ }^{51}$. Quant à Christine de Pizan, c'est aussi pour toucher aux racines du problème qu'elle transforme les calomnies des hommes critiquées dans La Cité des Dames en matière d'enseignement dans Trois Vertus. Pour répondre au jugement des hommes qui pensent que les femmes ne sont pas fidèles en amour ${ }^{52}$, Christine érige le principe suivant : « Le premier de ces -vii- poins et rigles que nous enseignons est que toute dame qui aime honneur, et semblablement toute femme estant en ordre de mariage, il apertient que 
elle aime son mary et vive en paix avec lui, ou autrement elle a ja trouvéz les tourmens d'enfer, ou n'a fors toute tempeste $»^{53}$; et si les femmes ne sont pas discrètes aux yeux des philosophes et écrivains ${ }^{54}$, voilà une leçon pour prévenir les femmes contre ce genre de problèmes et pour arriver ainsi à guider leurs pas: "Ci devise le quart enseignement de Prudence, qui est comment la saige princepce tendra discrete maniere [...] Ainsi la sage dame usera de ceste discrete dissimulacion et prudent cautele, [...] $»^{55}$. Quand les lois de moralité, chacune représentant un constituant de la "cage », sont établies dans Le Livre des Trois Vertus, elles peuvent former des femmes vertueuses de génération en génération comme ce que la poétesse a exprimé dans la dédicace à Marguerite de Bourgogne; et la qualité vertueuse des citoyennes de la cité des Dames forcera les misogynes à se taire et à arrêter leur diffamation sur le sexe opposé. Ces lois deviennent donc les remparts de la cité des Dames pour qu'elle puisse résister au temps et exister à jamais.

19 Si c'est le contexte historique, personnel et littéraire qui a donné naissance à Christine de Pizan en tant que femme écrivain, c'est l'œuvre de saint Augustin qui lui a fourni un moyen efficace de réaliser son ambition d'écrivain, celle de défendre les femmes. Ce que Lori Walters indique dans son article ${ }^{56}$ décrit bien l'influence de saint Augustin sur Christine de Pizan ainsi que la contribution de cette dernière à la continuation de la cause de l'auteur du De Civitate Dei : «L'œuvre de Christine, dont le titre fait écho à celui de son devancier, se présente comme le développement de ses projets mêmes. La Cité des Dames et sa suite, Le Livre des 'III· Vertus (1405) sont donc des moyens pour réaliser le rêve de saint Augustin sur terre ${ }^{57}$. Christine de Pizan a clairement compris, volontairement adopté et habilement appliqué la théorie de «deux cités » d'Aurélius Augustinus dans la création de ses deux livres représentatifs, La Cité et Le Trésor, et cet apprentissage doit être considéré comme un héritage - qui est plus lourd que l'emprunt du titre du De Civitate Dei - que la poétesse a reçu de son prédécesseur latin. Le rapport de ses deux livres montre bien que Christine a construit une cité des Dames sur le modèle de la cité de Dieu selon la forme : elle laisse sa narratrice s'adresser au Seigneur au début de $L a$ Cité des Dames, fait venir les trois Filles divines pour diriger la construction, installe les saintes dans les hautes tours de la cité, et surtout elle invite la Vierge Marie à dominer ce royaume féminin en tant que reine; aussi bien que selon le contenu : elle divise la communauté des femmes en "deux cités " tout en montrant à ses membres que la possibilité est égale pour chaque femme de devenir citoyenne de la cité des Dames. Cette intention de la poétesse française voit sa réalisation, c'est-à-dire la cité des Dames se confond avec la cité de Dieu lorsque Justice, la dernière des Déesses qui dirigent Christine dans la construction, finit son travail et lorsqu'elle appelle cette citadelle une cité de Dieu : Gloriosa dicta sunt de te civitas Dei ${ }^{58}$.

\section{NOTES}

1. Voilà ce que G. Bardy indique dans son introduction générale : « La plus ancienne traduction française est celle que Raoul de Praelles, avocat du roi (†1383), écrivit, avec des commentaires, pour Charles V, de 1371 à 1375 ». Euvres de Saint Augustin (33, 
cinquième série) : La Cité de Dieu (Livres I-V) : Impuissance sociale du paganisme (Texte de la $4^{\mathrm{e}}$ édition de B. Dombart et A. Kalb, introduction Générale et notes par G. Bardy, traduction française de G. Combès), Bruges, Desclée de Brouwer, 1959, p. 141.

2. Saint Augustin a ainsi parlé de la circonstance qui l'a poussé à écrire le De Civitate Dei : «Sur les entrefaites, Rome fut détruite sous les coups de l'invasion des Goths que conduisait le roi Alaric : ce fut un grand désastre. Les adorateurs d'une multitude de faux dieux, que nous appelons ordinairement les païens, s'efforcèrent de faire retomber ce désastre sur la religion chrétienne, et se mirent à blasphémer le vrai Dieu avec plus d'âpreté et d'amertume que d'habitude. C'est pourquoi, brûlé du “zèle de la maison de Dieu", je décidais d'écrire contre leurs blasphèmes ou leurs erreurs les livres de la Cité de Dieu ». (Saint Augustin Rétractations, traduction G. Bardy, BA, t. XXXIII, p. 167). Voir aussi l'introduction de G. Bardy : « Peut-être a-t-il fallu la prise de Rome par Alaric pour déterminer l'évêque d'Hippone à écrire l'œuvre à laquelle il songeait depuis longtemps déjà ", op. cit., p. 74.

3. Voir l'introduction de C. C. Willard à l'édition critique du Livre des Trois Vertus : " On a parfois dit que rien fut décidé par ce débat, mais Christine y gagna une certaine célébrité et devint par la suite l'avocate de la dignité féminine. Il est en effet évident que deux de ses ouvrages, et non des moindres, sont directement inspirés de l'affaire : Le Livre de la Cité des Dames et Le Livre des Trois Vertus, auquel on a parfois donné le titre du Trésor de la Cité des Dames. Les deux ouvrages furent composés vers 1405 ». Christine de Pizan, Le Livre des Trois Vertus, éd. C. C. Willard \& E. Hicks, Paris, Librairie Honoré Champion Éditeur, 1989, p. XI.

4. M. Zimmermann indique la même idée dans son article « Utopie et lieu de la mémoire féminine : La Cité des Dames » : «[...] : les deux œuvres appartiennent au genre de l'apologie, celle de saint Augustin défend le christianisme contre le reproche selon lequel les chrétiens seraient responsables de la chute de l'Empire Romain, tandis que Christine défend les femmes de l'accusation d'être un sexe inférieur et coupable de la perte du sexe masculin, [...] ». Au champ des escriptures, III Colloque international sur Christine de Pizan, Études réunies et publiées par E. Hicks, Paris, Honoré Champion Éditeur, 2000, p. 569.

5. « Pourtant, dans les vingt et un livres suivants, le thème de la destruction de Rome n'apparaît que très rarement, et toujours de façon épisodique. Dans les quelques jalons dont Augustin a marqué son œuvre et où, totalement ou partiellement, il la résume, il n'est presque jamais question de ce fameux sac de Rome ", J.-C. Guy, Unité et structure logique de la «Cité de Dieu » de saint Augustin, Paris, Études Augustiniennes, 1961, p. 6. Voir aussi à ce propos G. Bardy, op. cit. p. 21.

6. Saint Augustin, La Cité de Dieu, texte latin et traduction française avec une introduction et des notes par Jacques Perret, Paris, Librairie Garnier Frères, 1960, livre II, chapitre XXIX.

7. Selon saint Augustin lui-même, il traite le sujet de « deux cités » dans la deuxième partie de son œuvre, De Civitate Dei, en expliquant leurs origines, leurs développements ou leurs progrès, ainsi que leurs aboutissements respectifs. Voir, à ce propos, l'introduction de Jacques Perret pour la Cité de Dieu, op. cit., pp. VI-VII.

8. J'aimerais bien emprunter ici une citation à J.-C. Guy afin de pouvoir fournir une définition plus juste sur l'idée de « deux cités » de saint Augustin : « [...] tous les êtres, angéliques et humains, se distinguent selon leur référence à deux "cités" fondées sur deux amours ; ces deux cités, mélangées dans le temps de l'histoire, le Jugement 
dernier les séparera définitivement. Unité et structure logique de la «Cité de Dieu » de saint Augustin, Paris, Études Augustiniennes, 1961, p. 9.

9. Cité de Dieu, livre XIV, chapitre XXVIII.

10. Christine de Pizan, Le livre de la Cité des Dames, éd. M. C. Curnow, (The Livre de la Cité des Dames of Christine de Pisan : A Critical Edition), Vanderbilt University (thèse de Ph. D), 1975, p. 1035.

11. C. C. Willard, par exemple, exprime bien le rapport entre les deux livres de Christine de Pizan en disant que « The final chapter of The Book of the City of Ladies serves as an introduction to the second undertaking, The Book of the Three Virtues, which was designed to deal with certain problems of contemporary society and to prepare any woman who so desired to become a worthy inhabitant of the City of Ladies ». Christine de Pizan : Her Life and Works, New York, Persa Books, 1984, p. 145. Maureen Quilligan appelle aussi Le Trésor la suite de La Cité des Dames : «[...] locating the sequel to the Cité des dames - Le Trésor de la cité des dames - in the context of Christine's remonstrating letter to the queen and her general program for secular, [...] ». The Allegory of Female Authority: Christine de Pizan's Cité des Dames, Ithaca and London, Cornell University Press, 1991, p. 9. Sur ce même sujet, voir aussi mon article : « La communauté féminine : lien entre Le Livre de la Cité des Dames et Le Livre des Trois Vertus ", Romance Notes, vol. XXXIV, number 3, Spring, 1994, pp. 291-300.

12. Charity Cannon Willard n'hésite pas à appeler La Cité des Dames le premier volume des œuvres de cet écrivain en parlant des deux ouvrages rédigés après le Débat du Roman de la Rose: « The title of this first volum was inspired by Saint Augustine's City of God, [...] », Christine de Pizan : Her Life and Works, p. 135.

13. Au début du Livre des Trois Vertus, Christine la narratrice nous montre comment elle était forcée par les Trois Vertus de prendre la plume et de continuer à écrire après la construction de la Cité des Dames : « Aprés ce que j'oz ediffiee a l'ayde et par le commandement des troys Dames de Vertus, c'est assavoir Rayson, Droiture et Justice, La Cité des Dames par la forme et maniere que ou contenu de la dicte cité est declairié $[. .] »$, p. 7.

14. C. Lambot, « Lettre inédite de s. Augustin sur le De Civitate Dei », Revue bénédictine, tome 55,1939 , p. 112.

15. C'est la traduction de J.-C. Guy, op. cit., p. 12.

16. Je me permets d'emprunter ici l'argument de G. Bardy avancé dans son introduction générale, op. cit. : « Dans la réalité, il est permis de croire que saint Augustin avait depuis longtemps le désir d'écrire ce vaste ouvrage sur la cité de Dieu ou plus exactement sur les deux cités qui se partagent aujourd'hui l'empire du monde ». p. 20.

17. J.-C. Guy, op. cit., p. 7.

18. Ibid., p. 5.

19. «Or donc, de ces douze livres, les quatre premiers traitent de l'origine des deux cités, celle de Dieu, celle de ce monde ; les quatre suivants expliquent leurs développements ou leurs progrès; et les quatre derniers, les fins qui leur sont dues ». Cité de Dieu : Introduction, pp. VI-VII.

20. Cité des Dames, p. 618.

21. Ibid., p. 630.

22. Ibid., pp. 619-620.

23. Ibid. : « Hélas! Dieux, pourquoy ne me faiz tu naistre au monde en masculin sexe, a celle fin que mes inclinacions fussent toutes a te mieulx servir et que je ne errasse en riens et fusse de si grant parfeccion comme homme masle ce dit estre?», p. 621. 
24. «La cité est le groupement d'hommes de même origine, parlant la même langue, se serrant derrière les mêmes murailles, obéissant aux mêmes lois et adorant les mêmes dieux. Tout ce qui est en dehors, c'est l'étranger, l'ennemi, le barbare avec qui on n'entretient pas de relations ", G. Bardy, op. cit., p. 53.

25. Ibid., p. 57.

26. «Preudefame, par saint denis !/Dont il est mains que de phenis,/Si com valerius tesmoingne,/Ne puet nul amer qu'el nel poingne/De granz paours et de granz cures/Et d'autres mescheances dures ». Guillaume de Lorris et Jean de Meun, Roman de la Rose, Paris, Librairie Générale Française, 1992, p. 520.

27. Cité des Dames, p. 630.

28. Cité de Dieu, livre I, chapitre XXXV.

29. Voir la note 24 de cet article.

30. B. Ribémont, « De l'architecture à l'écriture : Christine de Pizan et La Cité des Dames ", La ville: du réel à l'imaginaire: Actes du Colloque de Rouen, 8-10 novembre 1988, textes rassemblés par J.-M. Pastré, Mont-Saint-Aignan, Publications de l'Université de Rouen, 1991, pp. 27-35.

31. «Aprés ce que j'oz ediffiee a l'ayde et par le commandement des troys Dames de Vertus, [...] », Christine de Pizan, Le Livre des Trois Vertus, op. cit., p. 7.

32. Ibid., p. 8.

33. Voir l'introduction de C.C. Willard (p. XII) et la dédicace de Christine de Pizan à la princesse de Bourgogne (pp. 3-4) dans Le Livre des Trois Vertus, op. cit.

34. Il s'agit ici du chapitre $\mathrm{X}$ de la deuxième partie du Livre des Trois Vertus.

35. G. Bardy, op. cit., p. 81.

36. Le Livre des Trois Vertus, p. 3.

37. Chapitre II de la première partie du Livre des Trois Vertus.

38. J.-C. Guy, op. cit. : « Il y est seulement question de ce qui deviendra un thème fondamental du De civitate Dei : tous les êtres, angéliques et humains, se distinguent selon leur référence à deux "cités" fondées sur deux amours ; ces deux cités, mélangées dans le temps de l'histoire, le Jugement dernier les sépare définitivement », p. 9.

39. Le Livre des Trois Vertus, pp. 10-11.

40. Ibid., p. 11.

41. Ibid., voir le chapitre VI.

42. G. Bardy, op. cit., p. 83

43. Livre XIV, chapitres 1-4 dans la Cité de Dieu ; chapitres II-VIII dans Le Livre des Trois Vertus.

44. Livre XIV, chapitres 15-22 dans la Cité de Dieu ; et chapitres XXII-XXVII dans Le Livre des Trois Vertus.

45. Livre XIX, chapitres 4-10 dans la Cité des Dieu ; et chapitre XXVII dans Le Livre des Trois Vertus.

46. J.- C. Guy, op. cit., p. 12.

47. Voir Willard, Christine de Pizan : Her Life and Works, op. cit., p. 135.

48. Willard a ainsi parlé du réalisme du livre du Trésor de la Cité des Dames dans son introduction à cet ouvrage : " C'est ainsi que Le Livre des Trois Vertus donne un tableau pittoresque et réaliste de la vie domestique à la fin du moyen âge, car en prévenant les femmes contre leurs défauts et faiblesses, il arrive à Christine d'évoquer des scènes tout aussi réalistes que celles des Quinze Joyes de mariage ou du Miroir de mariage d'Eustache Deschamps », p. XII. 
49. J.- C. Guy appelle la première partie de la Cité de Dieu la Réfutation et la secone la Démonstration. Voir son ouvrage, p. 12.

50. J.- C. Guy, op. cit., p. 13.

51. Ibid., p. 9.

52. «[...] plusieurs hommes dient que si pou soit de femme loyalles en la vie amoureuse », La Cité des Dames, p. 926.

53. Le Livre des Trois Vertus, p. 52.

54. « [...] et meesmement maistre Jehan de Meun trop l'afferme en son Rommant de la Rose, et autres autteurs aussi le font que homme ne die a sa femme chose que il vueille celer et que femmes ne se pueent taire ». (Cité, p. 843).

55. Le Livre des Trois Vertus, p. 61, 64.

56. L. Walters, « La réécriture de saint Augustin par Christine de Pizan : de la Cité de Dieu à la Cité des Dames ", Au champ des escriptures, op. cit., pp. 197-215.

57. Ibid., p. 214.

58. Le Livre de la Cité des Dames, p. 1031.

\section{AUTEUR}

\section{XIANGYUN ZHANG}

Emory \& Henry College, Virginie, USA 